\title{
Seguridad operacional de la pista del Aeropuerto Internacional Ramón Villeda Morales, hacia la certificación del aeródromo
}

\author{
Ramón Emilio Bueso \\ Iván Vladimir Betancourt
}

\section{Resumen}

Esta investigación es un aporte al fortalecimiento de la cultura de la Seguridad Operacional Aeroportuaria en uno de los cuatro aeropuertos internacionales con los que cuenta Honduras. Los Aeropuertos, según regulaciones internacionales, se esfuerzan en el cumplimiento de los estándares requeridos de Seguridad Operacional, estableciendo controles para la mitigación de los riesgos potenciales mediante la aplicación de estándares de Seguridad definidos por la Organización de Aviación Civil Internacional (OACl) en el Anexo 14 en lo referente al diseño y operaciones de aeródromos, y el Anexo de 19 (OACl) el cual trata en especial lo relativo a la implantación del Sistema de Gestión de Seguridad Operacional para prestadores de servicio de transporte aéreo, aeroportuario y de navegación aérea, entre los más importantes. Con la obtención de datos y una supervisión programada a la pista 04-22, junto con el equipo de certificación de la concesionaria Aeropuertos de Honduras (Interairports, S.A.) y el Departamento de Ciencias Aeronáuticas (DCA) de la Universidad Nacional Autónoma de Honduras (UNAH), se identificaron varios riesgos para implementar mejoras a la Seguridad Operacional en el Aeropuerto, por lo que, se identificó las zonas de la pista que no cumplen aún con las normativas nacionales e internacionales, los obstáculos presentes en el aeródromo que pueden generar un riesgo para las operaciones de las aeronaves, las áreas en los alrededores del aeródromo donde hay más presencia aviaria y fauna que podría generar en algún momento una incursión en pista.

Palabras clave: Seguridad Operacional, Aeropuerto, Certificación, Aeronave, Anexo $14 \mathrm{OACl}$, Anexo 19 OACl. 


\section{Abstract}

This article refers to research that is a contribution to strengthening to the culture of Aviation Safety, in one of the four international airports in Honduras. It is expected that the findings of this study encourage, the activation of mechanisms that the possibility of accidents/incidents decline during activities taking place in the areas of movement of an aerodrome, in this case referring to the track 04-22 of the International Airport Ramón Villeda Morales in La Lima, Cortés. The Airports according to international regulations strive to comply with the required standards of safety, establishing controls to mitigate potential risks by applying security standards defined by the International Civil Aviation Organization (ICAO) in Annex 14. With data collection and monitoring programmed to track 04-22, along with the certification team concessionaire Airports of Honduras (Interairports, SA) and the Department of Aeronautical Sciences (DCA) of the National Autonomous University of Honduras (UNAH), several risks were identified to implement improvements to safety at the airport, so that areas of the track that do not yet comply with national and international regulations identified, obstacles present at the airfield that can generate a daily risk to aircraft operations, the areas around the airfield where more bird and wildlife presence that could generate at some point a raid on track.

Keywords: TSafety Operations, Airport, Certification, Aircraft, Annex 14 (ICAO), Annex 19 (ICAO).

Ramón Emilio Bueso,(ramon.bueso@unah.edu.hn), Departamento de Ciencias Aeronáuticas, Universidad Nacional Autónoma de Honduras. Iván Vladimir Betancourt (iván.betancourt@unah.edu.hn), Departamento de Ciencias Aeronáuticas, Universidad Nacional Autónoma de Honduras. 


\section{INTRODUCCIÓN}

El presente artículo muestra los principales elementos de la investigación que se desarrolló para resaltar la importancia sobre el tema de la Seguridad Operacional, en este caso para la pista 04-22 del Aeropuerto Internacional Ramón Villeda Morales en La Lima, Cortés. La investigación buscó identificar las debilidades y riesgos que presenta en su entorno dicha pista bajo el análisis de los instrumentos utilizados durante este proceso de investigación, contribuyendo a la vigilancia para mantener la Seguridad Operacional en dicho aeródromo. Los instrumentos que se utilizaron inicialmente fueron entrevistas dirigidas específicamente al personal responsable del aeródromo y a expertos del área. A continuación, este proceso de investigación permitió realizar una inspección en la pista 04-22 del Aeropuerto Internacional Ramón Villeda Morales donde se utilizó una guía con preguntas formuladas y dirigidas a la aplicabilidad del cumplimiento de las normativas nacionales e internacionales establecidas, y que fueron discutidas junto con los encargados de la supervisión y mantenimiento del aeropuerto, logrando así la recolección de datos para explorar y describir la operatividad del aeródromo y a su vez encontrar las no conformidades en relación con las normas mencionadas que serán analizadas para el correcto análisis de riesgo.

La aplicación de los instrumentos de recolección generó suficiente información para identificar los tipos de riesgos que se podrían encontrar durante la inspección en la pista 04-22 del aeropuerto, lo cual favorecerá a las operaciones seguras de las aeronaves en la Gestión de la Seguridad Operacional.

Se consideró que esta investigación servirá de base para nuevas investigaciones relacionadas al campo aeronáutico, por ser de mucha importancia el conocer algunos de los puntos de riesgo que se localizan en la pista 04-22 del Aeropuerto Ramón Villeda Morales; así mismo agregar un valor al trabajo en equipo para la identificación y gestión de los riesgos operacionales en las pistas aeroportuarias, de esa manera crear un ambiente favorable para la realización de operaciones seguras de las aeronaves en los aeropuertos internacionales del País.

El presente artículo resalta la importancia que tiene el estudio de la Seguridad Operacional en un Aeropuerto, se describe la metodología que se utilizó, a través, de las consultas bibliográficas; de ellas se obtuvo información del marco regulatorio del campo aeroportuario sobre la aplicabilidad de las normas y procedimientos establecidos en las pistas de los Aeropuertos. Como parte de la investigación, el DCA se incorporó a una reunión con los diferentes actores involucrados en el proceso de certificación y seguidamente se procedió con una inspección de campo 
para la toma de datos en las áreas operativas de la pista 04-22 del Aeropuerto, en donde se logró hacer un levantamiento de información, por medio de entrevistas al personal responsable e inspecciones visuales. Se presentan los resultados que proponen identificar las áreas de mayor riesgo y que no cumplen con las normativas nacionales e internacionales, identificar en qué áreas de la pista hay más presencia aviaria, fauna; así como aquellas que necesitan de un mantenimiento correctivo; todo ello encaminado a dar la importancia que tiene la Seguridad Operacional en los Aeropuertos.

\section{METODOLOGÍA}

\section{Consultas bibliográficas}

La principal referencia utilizada para el desarrollo de la presente investigación fue un informe elaborado por el "Grupo Regional de Planificación e Implementación de la Organización de la Aviación Civil Internacional (OACI)", conocido por su nombre en inglés como Regional Planning and Implementation Group (GREPECAS/16 $\mathrm{NE} / 15)^{1}$. Este grupo examinó la aplicación de normas y métodos internacionales (SARP, por sus siglas en inglés), formulados por el Consejo de la Organización de Aviación Civil Internacional (OACI) en el año 2011, para los Aeropuertos regionales; este informe incluyó información relevante de la pista de aterrizaje y despegue del Aeropuerto Internacional Ramón Villeda Morales en la ciudad de la Lima Cortes.

Utilizando como referencia el Anexo $140 \mathrm{ACl}$, documento que contiene las normas y métodos recomendados (especificaciones) que prescriben las características físicas y las superficies limitadoras de obstáculos relativos al diseño y operaciones de los aeródromos (Anexo 14 Aerodromos, 2013).

Se utilizó como referencia Generalidades de Acrónimos y Definiciones y Abreviatura de las Regulaciones de Aeronáutica Civil RAC 139 que establece las normas que regulan la certificación y operación de aeródromos terrestres (Dirección General de Aeronáutica Civil, 2008).

\footnotetext{
${ }^{1}$ CAR/SAM Regional Planning and Implementation Group (GREPECAS) http://www2010.icao.int/SAM/Documents/GREPECAS/2011/GREPECAS16/Grp16Wp17Rev.pdf
} 
Se consultó la AIP (Publicación de Información Aeronáutica), lo cual facilito el conocimiento de información sobre las características físicas de la pista del Aeropuerto Internacional Ramón Villeda Morales.

El Anexo de 19 (OACI) Sistema de Gestión de Seguridad Operacional (SMS) que contiene procesos y sistemas destinados a la reducción del número de accidentes e incidentes que derivan la operación navegación aérea (Organización de Aviación Civil Internacional, 2013). Para tener información adicional del Aeropuerto Internacional Ramón Villeda Morales se utilizó como referencia el sitio web de Interairports S.A. (Aeropuertos de Honduras, 2015).

\section{Entrevistas}

Las entrevistas fueron dirigidas específicamente al personal responsable del aeródromo y a expertos del área.

\section{Visitas de Campo}

La visita de campo fue de suma importancia, lo que permitió realizar una inspección en las áreas de la pista 04-22 del Aeródromo junto con el personal de Aeropuertos de Honduras (Interairports, S.A.), autoridades de la Agencia Hondureña de Aeronáutica Civil (AHAC), consultores de la Organización de Aviación Civil Internacional $(\mathrm{OACl}$ ) y de Aviation Council International ( $\mathrm{ACl})$. Asimismo, se realizó una revisión y análisis de las normas internacionales y los métodos recomendados en el Anexo 14 al Convenio sobre Aviación Civil Internacional Diseño y Operaciones de Aeródromos, el RAC 139 Regulaciones de Aeronáutica Civil y su cumplimiento en dicho aeródromo.

Se aprovechó durante la observación de campo, para sostener una reunión con los diferentes actores, quienes son claves en el proceso de certificación del Aeropuerto Internacional Ramón Villeda Morales, de igual forma, junto con los consultores de los organismos Internacionales de la $\mathrm{OACl}$, personeros de la autoridad aeronáutica de Honduras, realizando así, una inspección general en puntos específicos de la pista 04-22 del aeropuerto para identificar los riesgos existentes. 


\section{Alcance del estudio}

A través de una investigación Descriptiva, considerando que con los estudios descriptivos se busca especificar las propiedades, las características y los perfiles de personas, grupos, comunidades, procesos, objetos o cualquier otro fenómeno que se someta a un análisis, se pretende medir o recoger información de manera independiente o conjunta sobre los conceptos o las variables a las que se refieren (Sampieri, 2014:124). Este

enfoque da a conocer la situación de la Seguridad Operacional de la Pista 04-22 del Aeropuerto Internacional Ramón Villeda Morales a la fecha en que se llevó a cabo la investigación, y así de esa manera, recomienda algunas alternativas de solución para el mejoramiento de la misma. Por tanto, la recolección de datos analizó los riesgos que pueden ocurrir por el no cumplimiento de las normas y métodos establecidos. Debemos hacer notar que, aunque la investigación no estaba dirigida a la calle de rodaje, sino a la pista 04-22, se aprovechó para incluirla en nuestro estudio como un lugar adyacente más de la pista 04-22.

\section{Tipo de análisis}

Con las opiniones y los datos recolectados según los instrumentos de investigación aplicados, se conocieron las no conformidades con relación a la normativa aeronáutica aplicable, en el supuesto, que el cumplimiento de la normativa establecida disminuye el riesgo en las operaciones en el aeropuerto estudiado.

\section{RESULTADOS}

La investigación generó varios resultados relevantes como ser: La constatación de las áreas de mayor riesgo operacional de la pista 04-22 del Aeropuerto Internacional Ramón Villeda Morales; la identificación de áreas de la pista donde puede haber discrepancia con las normativas nacionales e internacionales, incluyendo la existencia de obstáculos presentes en la pista del aeródromo y áreas en los alrededores del aeródromo, donde hay mayor presencia aviaria, de fauna y comunidades; la necesidad del mantenimiento en las áreas de la pista del aeropuerto, por elementos como: fisuras en calle de rodaje y daño de las luces empotradas en inicio de la pista 22 (algunos de estos elementos ya se encuentran en el programa de mantenimiento correctivo de la Concesionaria Aeropuertos de Honduras, Interairports S.A.). 
Situación actual del mantenimiento en la pista del Aeropuerto Ramón Villeda Morales

S Se constató que en el Aeropuerto Internacional Ramón Villeda Morales existen los procedimientos para proveer del mantenimiento a los indicadores de trayectoria de aproximación de precisión (PAPIS); además, cuenta con el

equipo calificado para su calibración, el que se realiza al menos tres veces al año, con lo cual, se previene el surgimiento de algún daño, lo cual garantiza su buen funcionamiento. Así mismo este sistema PAPI es calibrado por COCESNA una vez al año utilizando el avión verificador.

El aeropuerto cuenta con una programación para realizar las actividades propias para un correcto mantenimiento preventivo a las luces de pista; contando para tal, con el personal calificado para ejercer dicha función. Además, se conoció que cuando se suscitan las intervenciones correctivas estas se realizan oportunamente, sin que se pueda llegar a una situación de emergencia.

El pavimento de la pista 04-22 del Aeropuerto Ramón Villeda Morales se encuentra en general en buenas condiciones, según la inspección realizada durante la visita de campo y cuenta con el personal capacitado y entrenado para proveer de un mantenimiento periódico preventivo y correctivo.

Los márgenes de pista del Aeropuerto Ramón Villeda Morales no están definidos y por lo tanto no se cumplen con las normas nacionales y los SARPs (Standards and Recommended Practices) de la $\mathrm{OACl}$.

Se encontró que la franja de pista cumple parcialmente con las normas nacionales y los SARPs (Standards and Recommended Practices) de Seguridad de la $\mathrm{OACl}$, excepto lo que corresponde a las cercanías del lado este de la pista 04 donde se encuentran comunidades con alta población; todas estas tareas, están enmarcadas en el Programa de Mantenimiento Preventivo y Correctivo del concesionario.

El Aeropuerto Ramón Villeda Morales cuenta con un Runway End Safety Area (RESA) al extremo de la pista 04, cuya finalidad es la de reducir el riesgo de daños que pudieran ocasionar a una aeronave que efectúe un aterrizaje demasiado corto o un aterrizaje demasiado largo durante el recorrido de la pista de una aeródromo. (Anexo 14 Aerodromos, 2013). 
El aeropuerto cuenta con un programa y procedimientos documentados para la medición del coeficiente de fricción de la pista del aeropuerto, el cual es ejecutado por el supervisor de Seguridad Operacional y aprobado por el Gerente de Planificación.

Se tuvo conocimiento que el Aeropuerto Ramón Villeda Morales cuenta con un Programa de Supervisión y Vigilancia del área para evitar la producción de Objetos Extraños Escombros conocido como FOD (Foreign Object Debris); además, la patrulla móvil de seguridad del aeropuerto hace un recorrido de supervisión programado diariamente de identificación y recolección.

Los indicadores de dirección de viento están señalizados y en el emplazamiento adecuado, asimismo, cuenta con un programa de mantenimiento preventivo según las normas nacionales e internacionales establecidas por la $\mathrm{OACl}$.

Las señalizaciones horizontales / verticales en el pavimento de la pista del Aeropuerto Ramón Villeda Morales, se encuentran en buen estado.

\section{Áreas de mayor riesgo}

\section{Cultivos de Campesinos en zonas limítrofes al Aeropuerto}

Con la entrega de terrenos dentro del área concesionada y muy cercanos a la pista del Aeropuerto Ramón Villeda Morales por parte de Gobiernos anteriores a los grupos campesinos, a fin de incentivar la productividad agrícola; no obstante, dichos espacios ocasionan actualmente que la pista no se pueda expandir hacia el lado norte; además de contribuir a la atracción de aves, la generación de incendios como prácticas arcaicas de control de maleza para cultivos agrícolas y por ende aumentando el problema de la Seguridad Operacional.

Estos cultivos se presentan alrededor del boulevard de acceso al Aeropuerto y en el lado norte de la pista 22, donde las aeronaves hacen su aproximación instrumental utilizando el ILS (sistema de aterrizaje instrumental). Los peligros de estos cultivos cercanos al aeropuerto incluyen: generación de humo por las quemas trayendo consigo riesgos a las operaciones de las aeronaves; además, las aves buscan la manera de alimentarse aprovechando dichos cultivos cercanos a la pista del aeropuerto, también, sobrevuelan el aeropuerto haciendo incursiones en el espacio aéreo de la aeronave, de esa manera podrían impactar con ella y causar un incidente con daños a la aeronave, poniendo en riesgo la seguridad de vuelo. 


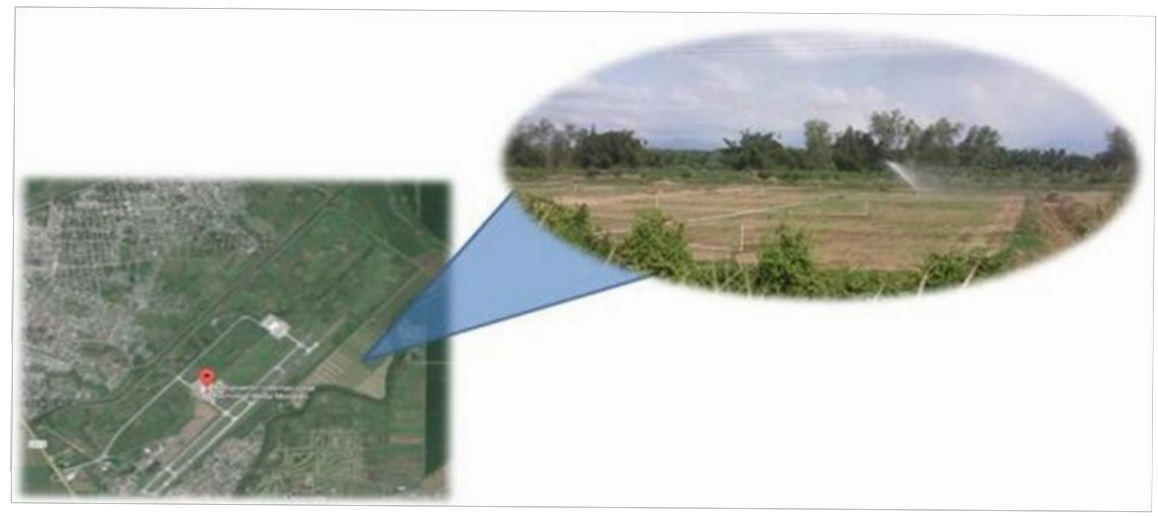

Figura 1. Cultivos de Campesinos en zonas limítrofes al Aeropuerto. Fuente: Google Maps.

\section{Casas de habitantes muy cerca al Aeropuerto}

Las casas de los habitantes de La Lima, Cortés se encuentran muy cerca de la pista del Aeropuerto, por lo que, se podría dar incursiones al muro perimetral, también, en una situación de emergencia la situación podría complicarse por la presencia de dichos habitantes pudiéndose verse afectados en el caso de cualquier incidente de las operaciones de las aeronaves en esa área.

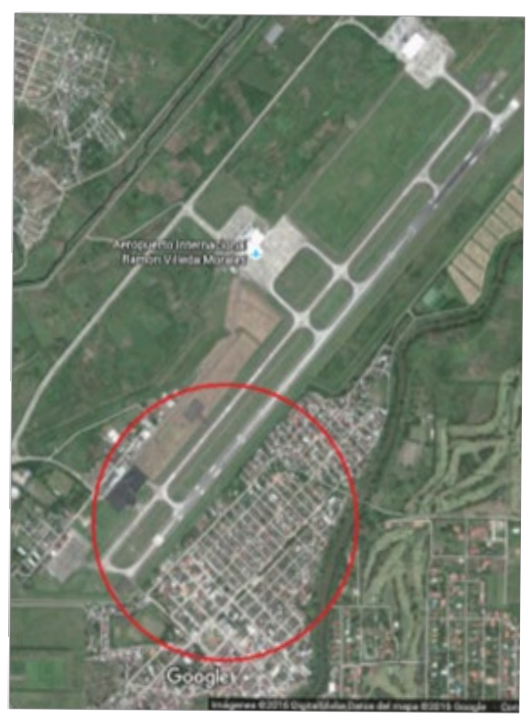

Figura 2. Extremo de la pista 04 muestra a habitantes de la lima en cercanías del Aeropuerto,

Fuente: Aeropuertos de Honduras (Interairports, S.A.) 


\section{Zonas de discrepancia con la normativa}

El extremo de la pista 22 del aeropuerto no cuenta con un área de seguridad de extremo de pista, conocido como RUNWAY END SAFETY (RESA) conforme la OACllo establece, que es una área simétrica respecto a la prolongación del eje de la pista y adyacente al extremo de la franja, cuyo objeto principal consiste en reducir el riesgo de daños a un avión que efectúe un aterrizaje demasiado corto o un aterrizaje demasiado largo.(Anexo 14 Aerodromos, 2013). También se logró detectar que la distancia entre el eje de la calle de rodaje y el eje de la pista del Aeropuerto Ramón Villeda Morales es menor que el estándar establecido por la $\mathrm{OACl}$ en su Anexo 14.

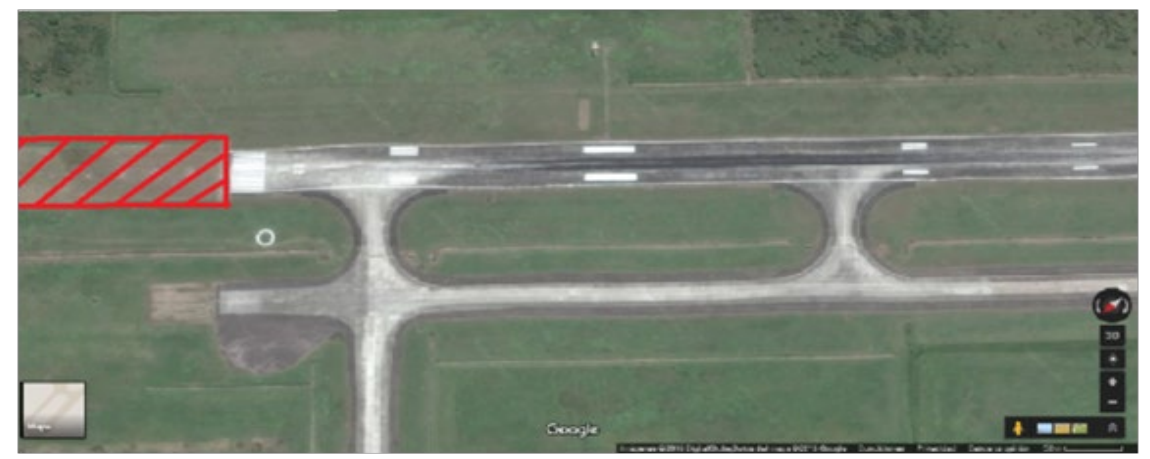

Figura 3. El extremo de la pista 22 mostrando ausencia de (RESA) RUNWAY END SAFETY AREA

\section{Obstáculos que generan riesgo}

Canales abiertos de Drenaje

Durante la inspección se pudo identificar la presencia de obstáculos en la franja de pista, en este caso los canales abiertos de drenajes; incumpliendo con la normativa que establece la $\mathrm{OACl}$ en relación con la zona libre de obstáculos de la franja de pista.Tomando un tercio de la pista 04-22 ubicados cerca del extremo 04 de la pista del Aeropuerto, se encontraron obstáculos en la franja de pista del Aeropuerto, que son los canales de drenaje los cuales no están preparados para soportar una aeronave que eventualmente tenga una excursión y pudiera llegar hasta ellos, así comoel bordo de control de inundaciones que se encuentra al lado este dentro de la franja de seguridad de pista. 




Figura 4. Especificaciones de Franja de Pista (Anexo 14 Aerodromos, 2013)

Siempre se debe verificar y asegurar que todos los drenajes no representen un obstáculo, ya que franja de pista está definida para reducir el riesgo de daños a las aeronaves en caso de que se salgan de la pista y proteger a las que la sobre vuelan durante las operaciones de despegue o aterrizaje. (Anexo 14 Aerodromos, 2013)

Presencia aviaria y fauna

Se identificó que los animales como ser: gatos, culebras, entre otros, que son capturados eventualmente, invaden el aeródromo. Se verificaron los espacios en el aeropuerto que pueden constituirse como refugio para la anidación de varios tipos de especies animal como ser aquellos lugares donde se almacena una cantidad de chatarra (ver imagen siguiente); estos espacios pueden ofrecer un descanso ya que brindan cierta protección del sol o la lluvia y esto suele ser atractivo para la fauna animal, que a su vez atrae agrandes predadores al lugar. Estos a su vez en su momento al morir, podrían atraer aves de rapiña (zopilotes) promoviendo el peligro aviario. 


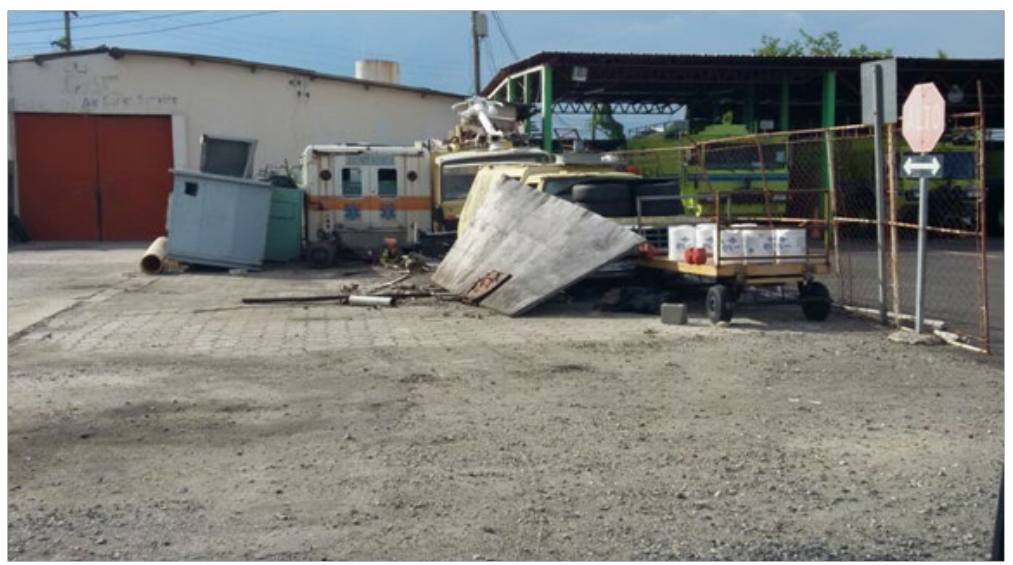

Figura 5. Acumulación de Chatarra Fuente: Foto tomada por equipo de investigación

En las inspecciones diarias realizadas en los perímetros del aeropuerto, como se observa en la imagen siguiente, la presencia de peligro aviario en zonas arboladas en los extremos de la pista del aeródromo es evidente, tal es el caso del zopilote negro, lo cual no significa que no haya presencia de otras especies de menor tamaño, que también representan un peligro aviario.

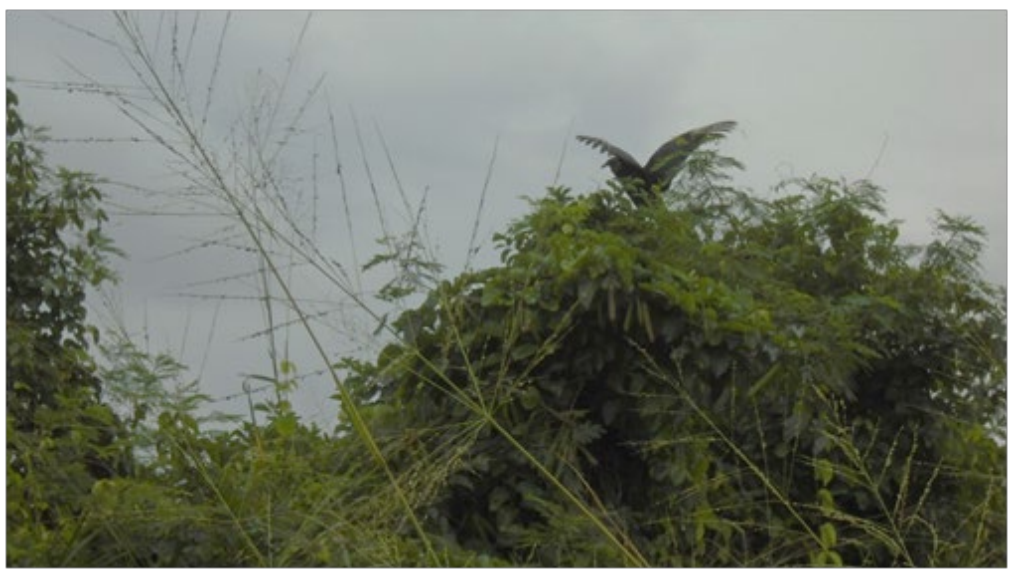

Figura 6. Presencia de Peligro aviario en los extremos de la pista 22, Fuente: Foto tomada por equipo de investigación

El coordinador de fauna del Aeropuerto Ramón Villeda Morales utiliza diferentes equipos y herramientas para ahuyentar en el momento que aparezca algún tipo de ave que sobrevuele las áreas de la pista del Aeropuerto. 


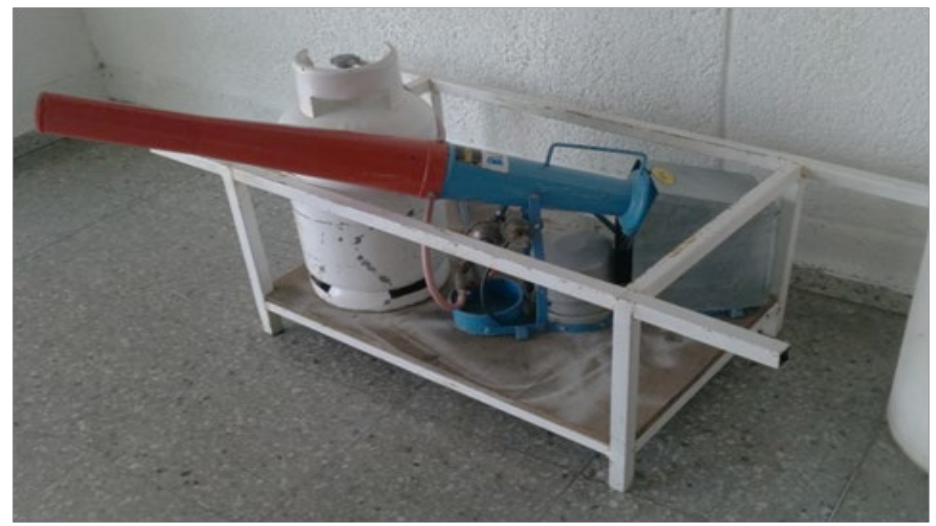

Figura 7. Cañón de gas propano para el Control de Avesen el Aeropuerto Ramón Villeda Morales Fuente: Aeropuertos de Honduras (Interairports, S.A.)

Necesidad de mantenimiento en áreas de la pista del aeropuerto

Fisuras en calle de rodaje

Como parte de los lugares adyacentes a la pista 04-22 se observó la calle de rodaje,en donde se pudo constatar pequeñas fisuras; no obstante, estas en su estado actual no representan un mayor riesgo para las operaciones del tránsito de las aeronaves; sin embargo, el no realizar el mantenimiento correspondiente, eventualmente resultaría en un empeoramiento de dichas fisuras. Vale destacar que el concesionario Aeropuertos de Honduras (Interairports, S.A.) en su Programa de Mantenimiento Preventivo y Correctivo contempla dichas reparaciones.

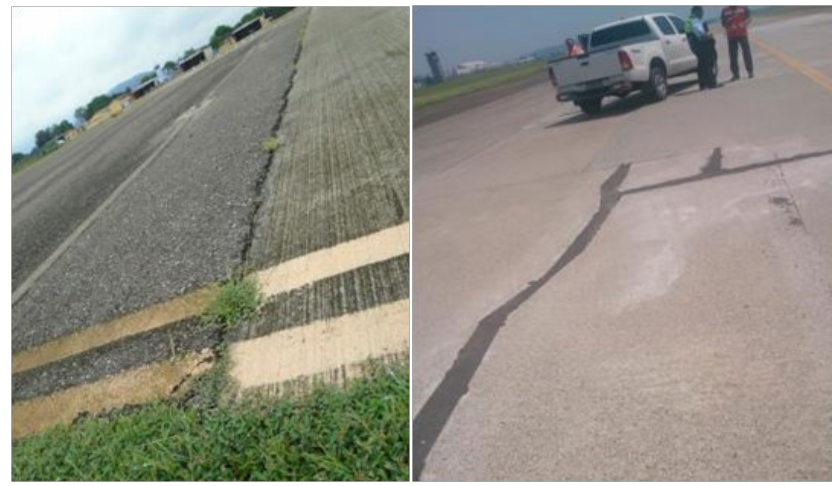

Figura 8. Presencia de fisurasen calle de rodaje (izq), pista en proceso de reparación (der) Fuente: Foto tomada por equipo de investigación. 
Mantenimiento en las luces empotradas en inicio de pista 22

Se observó durante la inspección en la pista 04-22 del Aeropuerto Ramón Villeda Morales, la necesidad de un mantenimiento correctivo donde se localizan las luces empotradas al inicio de la pista 22, actividad yaestipulada en el Programa de Mantenimiento del concesionario Aeropuertos de Honduras (Interairports, S.A.).

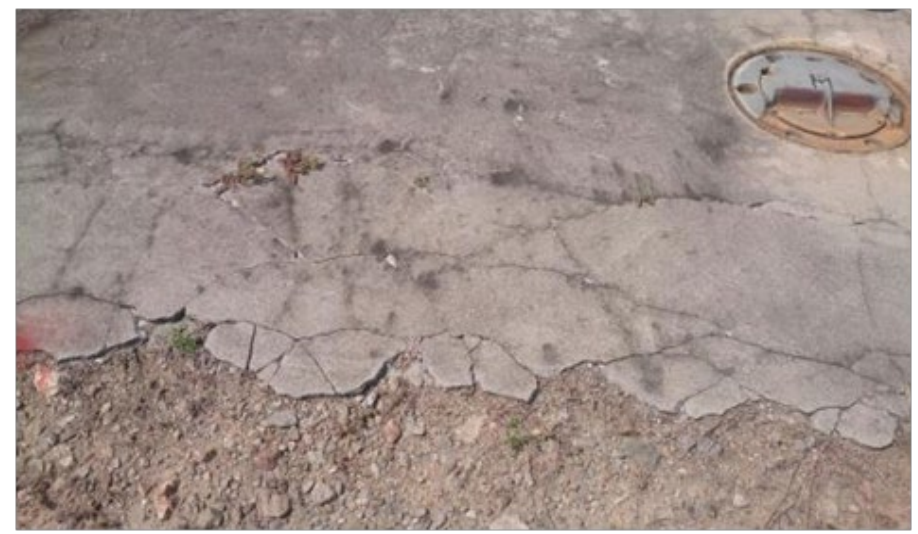

Figura 9. Luces empotradas en inicio de pista 22

Fuente: Foto tomada por equipo de investigación.

\section{DISCUSIÓN}

La realización de esta investigación pretende generar nuevas ideas para apoyo de futuras investigaciones sobre el estado operativo de las pistas en los aeródromos (ver recomendación 5), y que oriente al ánimo del trabajo en conjunto, necesario para identificar y gestionar las acciones correctivas a las no conformidades y así mitigar o eliminar los riesgos operacionales. Debido a que dentro de este sector aeronáutico, que es muy especializado, existen muy pocas investigaciones de este tipo en Honduras, la concesionaria Aeropuertos de Honduras (Interairports, S.A.) debe seguir trabajando en el estudio de este tipo de investigaciones, que le permitirán obtener resultados novedosos que favorezcan a los niveles aceptables de seguridad operacional en los aeródromos. Como todo estudio de investigación, se presentaron limitaciones debido a que la misma se contempló en el Aeropuerto Ramón Villeda Morales, para lo cual fue necesaria la movilización desde la ciudad de Tegucigalpa, Francisco Morazán hacia la ciudad de La Lima, Cortés. La visita de inspección se realizó en un tiempo muy limitado, con lo cual, no se pudo obtener un mayor número de datos para la investigación. 
El estudio de la Seguridad Operacional en los aeropuertos es muy importante, ya que busca identificar, prevenir o minimizar con suficiente anticipación el mayor número peligros a fin de evitar accidentes e incidentes derivados de la operación aérea. Esto se logrará mediante la mitigación o eliminación de las posibles amenazas encontradas o de incidentes menores sobre un sistema operativo, por lo que, es de suma importancia para los aeropuertos lograr un nivel aceptable de seguridad operacional, para evitar los riesgos operacionales y fomentar la motivación hacia una conducción segura de las operaciones diarias.

\section{CONCLUSIONES}

1. La Franja de Seguridad de Pista para un aeropuerto de Categoría 4D debe ser de 150 metros; en el caso del Aeropuerto Ramón Villeda Morales no cumple con este estándar, siendo esta de una dimensión menor. Esta no conformidad es más notoria en el extremo 04 en donde la anchura de la franja es mucho menor a la anchura de la franja del extremo 22 de la misma pista.

2. En términos generales, el entorno del Aeropuerto Ramón Villeda Morales cuenta con un ambiente relativamente rico en vegetación natural alta; este entorno natural en la pista 22 lleva a una mayor concentración de factores que pueden ser atractivos para la fauna con presencia en la zona; en este sector se cuenta con cultivos de semillas que pueden atraer distintos tipos de aves y sectores con mucha vegetación que pueden atraer diversos tipos de animales.

3. Como parte de los lugares adyacentes a la pista 04-22, se observó en la calle de rodaje pequeñas fisuras que en su estado actual no representan mayor peligro para el tránsito de aeronaves. A futuro si no se da el mantenimiento necesario a estas pequeñas fracturas del pavimento, eventualmente se dará un empeoramiento de dichas fisuras, lo cual, podría dañar en alguna medida los neumáticos y/o trenes de aterrizaje de una aeronave.

4. Durante la gira de observación se constató, dados los deterioros observados, que es necesario llevar a la práctica el mantenimiento preventivo programado de las señalizaciones horizontales de la pista de aterrizaje.

5. En la visita de campo a la pista y plataforma se observóen el pavimento de la pista, la existencia de señalizaciones horizontales y verticales, que en general se encuentran en buen estado, la existencia de un programa de mantenimiento de la pista, entre otros; dichos elementos dan cuenta del cumplimiento de estándares y regulaciones de la Autoridad Aeronáutica por parte de la 
concesionaria Aeropuertos de Honduras (Interairports, S.A.) para lograr la certificación del Aeropuerto Ramón Villeda Morales.

6. La distancia entre el eje de la calle de rodaje y el eje de la pista del Aeropuerto Ramón Villeda Morales es menor que el estándar establecido por la $\mathrm{OACl}$ en su Anexo 14, tabla 3.1, el cual es de 176 mts. para aeropuertos con clave de referencia 4D, clave que actualmente posee el Aeropuerto Ramón Villeda Morales para sus operaciones aeronáuticas.

\section{RECOMENDACIONES}

1. Es conveniente realizar periódicamente la chapia de la maleza; así como, proceder al retiro de la chatarra en el extremo 22 de la pista en donde existen mayores fuentes de atracción de animales que pueden generar peligro en las operaciones aeronáuticas.

2. En la calle de rodaje, dar seguimiento al mantenimiento de las áreas en donde una combinación de distintos factores habrían causado las pequeñas fisuras observadas, cuyo ensanchamiento puede generar daños operacionales a aeronaves.

3. Considerando que la franja de pista cumple la función de reducir el riesgo de daños a las aeronaves en la eventualidad de una excursión de pista, se recomienda verificar y asegurar que los canales de drenajes no representen un obstáculo que impida dicha función.

4. La recomendación 3.9.8 del Anexo 14 de $\mathrm{OACl}$ con respecto a Distancias mínimas de separación de las calles de rodaje (ver conclusión 6 anterior), establece que "pueden permitirse operaciones con distancias menores de separación de los establecidos en la tabla 3.1, en aeródromos ya existentes si un estudio aeronáutico indicara que tales distancias de separación no influirían adversamente en la seguridad, ni de modo importante en la regularidad de las operaciones de los aviones". Por lo tanto, se recomienda realizar un estudio aeronáutico usando como referencia el Apéndice 4 del Manual de Diseño de Aeródromos (Doc. OACI 9157), Parte 2; para garantizar la seguridad de las operaciones en el aeropuerto.

5. Se recomienda al Estado de Honduras hacer énfasis en la supervisión y vigilancia continua en todas las actividades de mantenimiento, para así contribuir al incremento de los niveles de seguridad operacional acordes con las 
normas y estándares nacionales e internacionales, con miras a la certificación del aeródromo.

\section{AGRADECIMIENTO}

Se agradece al concesionario Aeropuertos de Honduras (Interairports, S.A.) por haber colaborado en el presente trabajo de investigación desde el momento en que se concibió la idea hasta la elaboración del informe final, facilitando información y dando acceso al Aeropuerto Internacional Ramón Villeda Morales para recoger datos in situ. De igual forma se agradece a la Agencia Hondureña de Aeronáutica Civil (AHAC) por haber facilitado información oficial relacionada con la seguridad de las operaciones del Aeropuerto antes mencionado.

\section{BIBLIOGRAFÍA}

- Aeropuertos de Honduras. (2015). Aeropuertos de Honduras.

- Anexo 14 Aerodromos. (2013).

- Dirección General de Aeronáutica Civil. (2008). RAC 139. Tegucigalpa: DGAC.

- Organización de Aviación Civil Internacional. (2013). Anexo 19. Gestión de la Seguridad Operacional (Primera Edición ed.). 999 University Street, Montréal, Quebec, Canada H3C 5H7, Montréa, Canada: Organización de Aviación Civil Internacional.

- Sampieri, R. H. (2014). Metodología de la Investigación (Sexta Edidición ed.). Mexico D.F., Mexico: MCGRAW-HILL. 